\title{
SOME OPEN QUESTIONS IN THE THEORY OF SINGULARITIES ${ }^{1}$
}

\author{
BY OSCAR ZARISKI
}

ABstract. Three approaches to a theory of equisingularity of complex analytic (or algebraic) hypersurfaces are outlined, based respectively on topology (topological equivalence of embedded varieties), differential geometry (Conditions A and B of Whitney) and algebraic geometry (the author's inductive discriminant criterion). For each of these approaches some unsolved questions and (or) conjectures are formulated, especially in regard to the relationship between these three points of view.

1. If I were giving this address some 7 or 8 years ago, the open question which I would have then given top priority in my talk would have been the problem of reduction of singularities of algebraic varieties of dimension $>3$, over ground fields of characteristic zero, the case of surfaces and of three-dimensional varieties having been settled in some of my earlier work in the late thirties and the early forties, work which also included the solution of the general problem of local uniformization in characteristic zero, in any dimension (see [10], [11], [12] and [13]). For twenty years, after these earlier papers of mine had appeared in print, no further progress was made in the direction of the solution of the problem of reduction of singularities. Personally I felt that I have devoted enough time and effort to that problem, that I needed a change of pace, and have therefore turned to other questions in my field. Some of my mathematical friends believed, no doubt, that during these twenty years I never gave up trying, and it is quite possible that these friends have drawn the-to me flattering-conclusion that since I am not able to prove the general reduction theorem, that theorem must be false. It is even probable that they were greatly tempted to look for and find a counterexample. Fortunately, Hironaka put a stop to this state of affairs by his

1 Retiring Presidential address delivered before the Seventy-seventh Annual Meeting of the Society in Atlantic City on January 22, 1971; received by the editors February 11, 1971; the author wishes to thank the NSF for support under the Grant No. GP-9667 to Harvard University.

AMS 1970 subject classifications. Primary 14B05, 32C40; Secondary 14H20, $32 \mathrm{C} 45$.

Key words and phrases. Singularity, hypersurface, topological equisingularity, differential-geometric equisingularity, algebro-geometric equisingularity, equimultiplicity, blowing-up transformation, equisingularity along a subvariety, Whitney stratification, equisingular projection, equisaturation. 
fundamental paper published in 1964 [4]. Thus that general problem was settled, at last, in the affirmative.

There still remains, however, the unsolved problem of reduction of singularities in characteristic $p \neq 0$. Whatever progress was made in this problem is associated primarily with the name of one single man, and that is Abhyankar. (Also some recent work of Hironaka, some of it unpublished, has contributed significantly to the problem in the case $p \neq 0$.) In his dissertation, published in 1956 [1], Abhyankar solved this problem for surfaces over perfect ground fields. Ten years later he extended his proof to arithmetic surfaces [2], i.e., to surfaces defined over a Dedekind domain, instead of over a field. Using various algorithms devised by him in the case of characteristic $p \neq 0$ he was also able to extend to that case [3] my proof of reduction of singularities of embedded surfaces, and therefore also my original proof of the birational reduction of singularities of three-dimensional varieties, under the assumption that $p<3$ !, i.e., $p \neq 2,3,5$. This is the state of the problem at present. Even the problem of local uniformization in characteristic $p \neq 0$ is still unsolved in dimension $>3$ (and also in dimension 3 , if $p=2,3,5)$.

2. What I wish to discuss here today is not this open question of how to get rid of singularities in characteristic $p \neq 0$, but rather the one of how to classify singularities in characteristic zero, in fact-and more specifically-in the complex domain. In recent years there was an upsurge of interest in the study of singularities of algebraic, or-more generally - of complex-analytic varieties. The most substantial contributions here were made by differential topologists rather than by algebraic geometers. The fields of topology and differential geometry have already in their possession a number of powerful tools for the exploration of the structure of the neighborhood of a singular point of a complex-analytic variety. On the other hand, the purely algebraic approach, while still in its infancy, seems to be the most natural approach to the subject, for it is doubtful whether singularities of complex-analytic varieties are purely topological or even differentialgeometric phenomena.

I will restrict myself to singular points $P$ of hypersurfaces $V_{r}$ :

$$
V_{r}: f\left(x_{1}, x_{2}, \cdots, x_{r}, x_{r+1}\right)=0, \quad P \in V_{r} \subset A_{r+1},
$$

i.e., to singular points $P$ of $r$-dimensional varieties $V_{r}$ such that, locally at $P, V_{r}$ can be embedded in a complex affine $(r+1)$-space $A_{r+1}$. Hence, locally at $P, V_{r}$ can be defined by the single equation (1), where $f$ is a convergent power series and $P$ is the origin. The case of hypersurfaces is the most interesting one, and is, of course, also the 
one which is relatively easier to handle (see also footnote 2 ). Now, suppose we have another hypersurface $V^{\prime}$ of the same dimension $r$, and a singular point $P^{\prime}$ on it. The basic question is the following: what shall we mean by saying that the two singularities $P, P^{\prime}$ are equivalent? The relation of equivalence which we are trying to spell out and which we shall designate by the term "equisingularity" should formalize our vague and not very intuitive idea of singularities of the same type, of the same degree of complexity. One thing is clear: it must be an equivalence relation which is much weaker than an analytical isomorphism.

Topology provides one possible answer which has the great advantage of being clear-cut and unambiguous: $V_{r}$ and $V_{r}^{\prime}$ are equisingular at $P$ and $P^{\prime}$ if they are topologically equivalent, as embedded varieties, in the neighborhood of $P$ and of $P^{\prime}$, i.e., if there exists a local homeomorphism $f: \boldsymbol{A}_{r+1} \rightarrow \boldsymbol{A}_{r+1}^{\prime}$ of the ambient affine spaces of $V_{r}$ and $V_{r}^{\prime}$ which sends $P$ into $P^{\prime}$ and $V_{r}$ into $V_{r}^{\prime} .^{2}$

This definition raises immediately a number of questions. I shall formulate here only two questions, which, as far as their degree of complexity is concerned, are respectively on the relatively shallow and the relatively deep end of a whole spectrum of possible questions. If the answer to the easier question cannot be provided by topologists in a relatively short order, I would be greatly disappointed. On the other hand, I would not blame them if they found it difficult to answer the second question.

The simplest numerical character of a singular point $P$ of $V$ is its multiplicity $e$, i.e., the degree of the leading form of the power series $f: e=e(V, P)$. Here $e=1$ if and only if $V$ is an analy tic manifold at $P$. Any definition of equisingularity should imply equimultiplicity, at the very least. Thus our first question is the following:

A. Does topological equisingularity of $V_{r}$ and $V_{r}^{\prime}$ at $P$ and $P^{\prime}$ imply that $e\left(V_{r}, P\right)=e\left(V_{r}^{\prime}, P^{\prime}\right)$ ?

The answer is known to be in the affirmative in the case of curves $(r=1)$. For $r>1$, I find in Milnor's account on singularities of complex hypersurfaces a general theorem $[6$, p. 5] from which an affirmative answer can be deduced in the following case: $e\left(V_{r}, P\right)=1$, and $P^{\prime}$ is at worst an isolated singular point of $V_{r}^{\prime}$.

The second question is the following:

We apply to the affine space $\boldsymbol{A}_{r+1}$ a locally quadratic transformation with center $P$, or a blowing-up transformation, whose effect

2 This definition would definitely be out of order for varieties which are not hypersurfaces. For instance, it is not difficult to see that any two algebroid branches $V_{1}$ and $V_{1}^{\prime}$ in $A_{n}, n \geqq 3$, are topologically equivalent as embedded varieties (of real dimension 2). 
can be informally described by saying that we remove the point $P$ from the affine space and we replace it by a projective $r$-space $P_{r}$ whose points represent the directions about $P$. The result is a nonsingular $(r+1)$-dimensional manifold $\bar{M}_{r+1}$ and a continuous surjective map

$$
T: \bar{M}_{r+1} \rightarrow A_{r+1}
$$

such that

$$
T^{-1}\{P\}=\bar{E}_{r} \subset \bar{M}_{r+1}, \quad \bar{E}_{r} \cong P_{r},
$$

and such that the restriction of $T$ to $\bar{M}_{r+1}-\bar{E}_{r}$ is an analytic isomorphism:

$$
\bar{M}_{r+1}-\bar{E}_{r} \stackrel{\sim}{\rightarrow} A_{r+1}-P .
$$

There is then a unique $r$-dimensional variety $\bar{V}_{r} \subset \bar{M}_{r+1}$ defined by the two conditions

$$
T\left(\bar{V}_{r}\right)=V_{r}, \quad \bar{E}_{r} \nsubseteq \bar{V}_{r} .
$$

The variety $\bar{V}_{r}$ is sometimes referred to as the proper transform of $V_{r}$. In view of the inclusion $\bar{V}_{r} \subset \bar{M}_{r+1}$, this variety is locally a hypersurface at each of its points. Let

$$
\bar{\varepsilon}_{r-1}=\bar{E}_{r} \cap \bar{V}_{r}
$$

The variety $\varepsilon_{r-1}$ is pure $(r-1)$-dimensional; it is the inverse image, on $\bar{V}_{r}$, of the point $P$, and is called the exceptional variety created by our blowing-up transformation of $V_{r}$. Again, also $\bar{\varepsilon}_{r-1}$ is locally a hypersurface, in view of the inclusion $\bar{\varepsilon}_{r-1} \subset \bar{E}_{r}$. Thus the total effect of our transformation leads from the pair $\left(V_{r}, P\right)$ to the pair $\left(\bar{V}_{r}, \bar{\varepsilon}_{r-1}\right)$.

Let us operate by a similar transformation on the pair $\left(V_{r}^{\prime}, P^{\prime}\right)$, getting a pair $\left(\bar{V}_{r}^{\prime}, \overline{\mathcal{E}}_{r-1}^{\prime}\right)$ consisting of the proper transform $\bar{V}_{r}^{\prime}$ of $V_{r}^{\prime}$ and of the exceptional $(r-1)$-dimensional subvariety $\bar{\varepsilon}_{r-1}^{\prime}$ which corresponds to the point $P^{\prime}$. Our second question is the following:

B. Prove that there exists a homeomorphism $f: \overline{\mathcal{E}}_{r-1} \rightarrow \overline{\mathcal{E}}_{r-1}^{\prime}$ such that if $\bar{P}^{\prime}=f(\bar{P}), \bar{P} \in \overline{\mathcal{E}}_{r-1}$ then $(1)\left(\overline{\mathcal{E}}_{r-1}, \bar{P}\right)$ and $\left(\overline{\mathcal{E}}_{r-1}^{\prime}, \bar{P}^{\prime}\right)$ are topologically equisingular and (2) $\left(\bar{V}_{r}, \bar{P}\right)$ and $\left(\bar{V}_{r}^{\prime}, \bar{P}^{\prime}\right)$ are also topologically equisingular.

Again the answer to this question is in the affirmative for $r=1$, showing that the classical algebro-geometric notion of equivalence of singularities of plane algebraic (or algebroid) curves, which goes back to Max Noether (see, for instance [14]), coincides with the topological concept of equivalence. (Note that if $r=1$ then both ( $\overline{\mathcal{E}}_{r-1}$ and $\overline{\mathcal{E}}_{r-1}^{\prime}$ are finite sets of points.) 
3. Let me now take a new tack which promises a better wind. Instead of dealing with a pair of hypersurfaces, let us consider analytic families of hypersurfaces $V_{r}$, all having a singular point at the origin and depending on a set of parameters $(t)=\left(t_{1}, t_{2}, \cdots, t_{s}\right)$. Thus, the variable member of this family will be a hypersurface $V_{r}^{(t)}$ defined by an equation

$$
V_{r}^{(t)}: f\left(x_{1}, x_{2}, \cdots, x_{r}, x_{r+1} ; t_{1}, t_{2}, \cdots, t_{s}\right)=0,
$$

where $f$ is a convergent power series in $r+s+1$ variables $(x),(t)$, in the neighborhood of $(x)=(t)=0$, and where

$$
f\left(0,0, \cdots, 0,0 ; t_{1}, t_{2}, \cdots, t_{s}\right) \equiv 0 \text {, identically. }
$$

Equation (2) can also be interpreted as the equation of a hypersurface

$$
V_{n}: f((x) ;(t))=0, \quad n=r+s, V_{n} \subset A_{n+1} .
$$

This hypersurface carries the nonsingular manifold

$$
W: x_{1}=x_{2}=\cdots=x_{r+1}=0 ; \quad r=\operatorname{cod}_{V_{n}} W .
$$

Our family of $r$-dimensional hypersurface $V_{r}^{(t)}$ now appears as a family of sections $V_{r}^{(\bar{t})}$ of $V_{n}$, transversal to $W$ :

$$
V_{r}^{(\bar{t})}=V_{n} \cap\left(t_{j}=\bar{t}_{j}, j=1,2, \cdots, r\right),
$$

the singular point of $V_{r}^{(\bar{l})}$ being the point

$$
P^{(\bar{t})}:\left(0,0, \cdots, 0, \bar{t}_{1}, \bar{t}_{2}, \cdots, \bar{t}_{s}\right) .
$$

We are interested particularly in the initial zero values of the parameters $t$, and therefore in the particular pair $\left(V_{r}^{(0)}, P^{(0)}\right)$, which we shall denote by $\left(V_{r}, P\right)$.

DEFINITION 1. $V_{n}$ is topologically equisingular (top. eqs.) at $P$, along $W$, if $\left(V_{r}^{(\bar{t})}, P^{(\bar{t})}\right)$ and $\left(V_{r}, P\right)$ are topologically equisingular, for all $(\bar{t})$ sufficiently near zero.

Following Whitney [8] and [9], one introduces two differentialgeometric conditions $\mathrm{A}$ and $\mathrm{B}$ which may or may not be satisfied by the triplet $\left(V_{n}, W, P\right)$ :

Condition A. If $P=\lim P_{i}$, where the $P_{i}$ are simple points of $V_{n}$, and if $T_{0}=\lim T\left(V_{n}, P_{i}\right)$, then $T_{0} \supset T(W, P)$.

Here $T\left(V_{n}, P_{i}\right)$ denotes the tangent space of $V_{n}$ at $P_{i}$, and similarly for $T(W, P)$.

Condition B. If $P=\lim P_{i}$ (as in $\left.\mathrm{A}\right)$, if also $P=\lim Q_{i}$, where the $Q_{\imath}$ are simple points of $W\left(P_{i} \neq Q_{i}\right)$ and if $\lim P_{i} Q_{i}=l_{0}$, and $\lim T\left(V_{n}, P_{i}\right)$ $=T_{0}$, then $l_{0} \subset T_{0}$.

It can be proved that both conditions are intrinsic, i.e., they are 
independent of the embedding of $V_{n}$ in affine $(n+1)$-space.

Definition 2. $V_{n}$ is differentially equisingular (diff. eqs.) at $P$, along $W$, if both Conditions $\mathrm{A}, \mathrm{B}$ are satisfied by $\left(V_{n}, W, P\right)$.

Whitney has proved that given any subvariety $W$ of $V_{n}$, the set of points

$$
\bar{Z}\left(V_{n}, W\right)=\left\{P \in W \mid V_{n} \text { is not diff. eqs. at } P \text { along } W\right\},
$$

is contained in a proper analytic (or algebraic, if $V_{n}$ and $W$ are algebraic varieties) subvariety of $W$. This allows him to define a stratification of $V_{n}$ (called the Whitney stratification) with the property that $V_{n}$ is differentially equisingular along each stratum, at each point of the stratum. If $r>1$ it is not known whether the answer to the following question is in the affirmative:

C. Is $\bar{Z}\left(V_{n}, W\right)$ a subvariety of $W$ ?

If $r=1$, the answer is known to be in the affirmative (see, for instance, Zariski [15]).

The deepest result obtained so far in the theory of equisingularity is the following result, due to Thom and proved by Thom [7] and later also by Mather:

If $S$ is any stratum of a Whitney stratification of $V_{n}$ then $V_{n}$ is topologically equisingular along $S$, at each point of $S$.

We note explicitly that this result does not signify that diff. eqs. of $V_{n}$ at $P$, along a subvariety $W$ (having $P$ as a simple point) implies top. eqs. of $V_{n}$ at $P$, along $W$. This latter statement may actually be false. For instance, if $a, b, c$ are integers such that $1<a \leqq \min \{b, c\}$, then the singular locus of the surface $V_{2}: z^{a}=x^{b} y^{c}$ consists of the two lines $W_{1}: x=z=0$ and $W_{2}: y=z=0$. For each $i=1,2$ and for each point $Q$ of $W_{i}$, the triplet $\left(V, W_{i}, Q\right)$ satisfies both Conditions $\mathrm{A}$ and B. In particular, $V_{2}$ is therefore diff. eqs. at the origin $P$ along $W_{i}$ $(i=1,2)$. However, while any section of $V_{2}$, transversal to $W_{i}$, at any point $Q$ of $W$, different from $P$, has at $Q$ an ordinary double point (i.e., a node), no section of $V_{2}$, transversal to $W_{i}$, at $P$ has a singularity at $P$ as simple as a node. Thus $V_{2}$ is not top. eqs. at $P$, along $W_{i}(i=1,2)$. Since in a Whitney stratification any two strata must be disjoint and the boundary of any stratum must be a union of strata, the Whitney stratification of our surface $V_{2}$ must consist of the following four strata: $V_{2}-\left(W_{1} \cup W_{2}\right), W_{1}-P, W_{2}-P, P$. We thus see that the strata of a Whitney stratification of $V_{n}$ are not necessarily maximal sets of differential equisingularity of $V_{n}$; in other words, it may very well happen for some stratum $S$ that $V_{n}$ is diff. eqs. along the closure $\bar{S}$ of $S$ at some point $P$ of the boundary $\bar{S}-S$ of $S$.

One may ask whether the converse of Thom's result is true: 
D. Does topological equisingularity imply differential equisingularity?

I have proved that the answer is in the affirmative if $r=1$. (We recall that $r$ is the codimension of $W$ on $V_{n}$.)

One may cite at this point also the following result, due to Hironaka [5]:

Differential equisingularity of $V_{n}$ at $P$, along $W$, implies equimultiplicity of $V_{n}$ at $P$, along $W$.

This result should be compared with the content of Question A.

4. I now come to the last part of my talk, in which I would like to describe briefly my own attempt to define equisingularity in a purely algebraic fashion. While this attempt was completely successful in codimension 1, in higher codimension I have now, by and large, only questions and conjectures. I may say, however, that my attempt to deal with the general case did pay me some dividends, since it led me to my general theory of saturation of local rings of singular points, a theory which I have initiated in 1968 [16] and which will be further developed in a series of papers, some of which are in course of publication and some in preparation.

Let the parameters $t_{1}, t_{2}, \cdots, t_{s}$ in (3) be now denoted by $x_{r+2}$, $x_{r+3}, \cdots, x_{n+1}$, where $n=r+s$. Then $V\left(=V_{n}\right)$ is defined by

$$
V: f\left(x_{1}, x_{2}, \cdots, x_{n}, x_{n+1}\right)=0,
$$

and $P$ still denotes the origin. Consider a set of $n$ elements $z_{1}, z_{2}$, $\cdots, z_{n}$ of the local ring of $V$ at $P$ :

$$
z_{i}=z_{i}\left(x_{1}, x_{2}, \cdots, x_{n+1}\right)=z_{i, 1}+z_{i, 2}+\cdots, \quad i=1,2, \cdots, n,
$$

where the $z_{i}$ are convergent power series in the $x$ 's, and $z_{i, \alpha}$ is homogeneous, of degree $\alpha$.

We shall say that the $n$ elements $z_{i}$ form a set of parameters if the following two conditions are satisfied:

(a) $(x)=0$ is an isolated solution of the $n+1$ equations

$$
z_{1}(x)=z_{2}(x)=\cdots=z_{n}(x)=f(x)=0 .
$$

(b) The $n$ linear forms $z_{i, 1}$ are linearly independent.

If condition (b) is satisfied, then the $n$ linear equations

$$
z_{i, 1}(x)=0, \quad i=1,2, \cdots, n,
$$

define a line $l_{z}$ through $P$. If that line does not belong to the tangent cone of $V$ at $P$, then condition (a) is automatically satisfied, and the $z_{i}$ are parameters. We say in this case that the $z_{i}$ are transversal parameters.

If the $z_{i}$ are parameters, then the $n$ equations $z_{i}(x)=0, i=1,2$, 
$\cdots, n$, define a regular curve $\Gamma$ through $P$ whose tangent line is $l_{z}$, and $P$ is an isolated intersection of $\Gamma$ and $V$. Let $m$ be the intersection multiplicity of $\Gamma$ and $V$, at $P$ :

$$
m=i(V, \Gamma ; P) \text {. }
$$

Here $m \geqq e(V, P)$, with equality if and only if the $z_{i}$ are transversal parameters.

The $n$ parameters $z_{i}$ define a projection $\pi_{z}$ of $V$ onto a neighborhood of the origin of the affine $n$-space of the $n$ variables $z_{i}$ :

$$
\pi_{z}: V \rightarrow A_{n}, \quad \pi_{z}\left(\bar{x}_{1}, \cdots, \bar{x}_{n+1}\right)=\left(z_{1}(\bar{x}), z_{2}(\bar{x}), \cdots, z_{n}(\bar{x})\right) .
$$

The direction of the line $l_{z}$ above is called the direction of the projection. We call the projection $\pi_{z}$ transversal if the parameters $z_{i}$ are transversal.

The full inverse image $\pi_{z}^{-1}(\bar{z})$ of any point $\bar{z}$, near zero, consists of at most $m$ points. The set of critical points $\bar{z}$, for which this full inverse image consists of less than $m$ points, is a hypersurface in $\boldsymbol{A}_{n}$, which we shall denote by $\Delta_{z}$; it is the critical variety of the projection $\pi_{z}$. The equation $D_{0}(z)=0$ of $\Delta_{z}$ can be obtained by forming the discriminant $D$ of a suitable polynomial, of degree $m$, with coefficients which are power series in the $z_{i}$, and by letting $D_{0}(z)$ be the product of the distinct irreducible factors of $D$.

Now, let $W$ be a subvariety of $V$, of codimension $r$, having at $P$ a simple point. We shall say that the projection $\pi_{z}$ is permissible, ormore precisely-W-permissible, if the line $l_{z}$ (the direction of $\pi_{z}$ ) is not contained in $T(W, P)$. Note that each transversal projection is $W$-permissible, since $T(W, P) \subset$ tangent cone of $V$.

Let $\pi_{z}$ be a permissible projection. Then $\pi_{z}(W)$ is a nonsingular variety $\bar{W}$, of the same dimension as $W$, with a simple point at $\bar{P}$ $=\pi_{z}(P)$. Since we assume that $W$ is a singular subvariety of $V$, we have $\bar{W} \subset \Delta_{z}, \operatorname{cod}_{\Delta_{z}} \bar{W}=r-1$, and we are dealing with a triple $\left(\Delta_{z}, \bar{W}, \bar{P}\right)$, in codimension $r-1$.

If $r=1$, then $\operatorname{cod}_{\Delta_{z}} \bar{W}=0$, i.e., $\bar{W}$ is an irreducible component of $\Delta_{z}$. In that case, equisingularity of $\Delta_{z}$, at $\bar{P}$, along $\bar{W}$, means simply that $\bar{P}$ is a simple point $\Delta_{z}$. I have proved in 1965 the following if $r=1$ (see [15]):

(1) If there exists a permissible projection $\pi_{z}$ such that $P$ is simple for $\Delta_{z}$, then $V$ is equisingular at $P$, along $W$.

(2) Conversely, if $V$ is equisingular at $P$, along $W$, then $\bar{P}$ is simple for $\Delta_{z}$ for any permissible transversal projection $\pi_{z}$.

I therefore was led to the following inductive definition of equisingularity: 
DEFINITION 3. $V$ is (algebro-geometrically) equisingular at $P$, along $W$, if there exists a permissible projection $\pi_{z}$ such that $\Delta_{z}$ is equisingular at $\bar{P}$, along $\bar{W}$.

With this definition of equisingularity certain statements which are not obvious or even not known to be true for topological or differential equisingularity, become either straightforward consequences or can be proved inductively without too much difficulty. For instance, we can assert the following:

1. Equisingularity of $V$ at $P$, along $W$, implies equimultiplicity of $V$ at $P$, along $W$.

2. The set $\operatorname{Eqs}(V, W)$ of points $P$ of $W$ such that $V$ is equisingular at $P$ along $W$, is the complement of a proper analytic (or algebraic) subvariety of $W$.

(Compare Result 2 with the unsolved Question C).

These results allow us to define a stratification of $V$ such that $V$ is equisingular along each stratum, at each point of the stratum.

The following is an open question:

If $P$ is a point on the boundary of a stratum $S$, it is true then that $V$ is not equisingular at $P$, along the closure of $S$.

The basic open question is the following:

E. Does algebro-geometric equisingularity imply topological equisingularity or even differential equisingularity?

Of course, in codimension 1 we know that all the 3 types of equisingularity are essentially identical concepts.

Assuming equisingularity of $V$ at $P$, along $W$, an even more important question, from an algebro-geometric point of view, is the one which presents itself naturally when we apply to the ambient affine space $\boldsymbol{A}_{n+1}$ of $V$ a blowing-up transformation $T$ with center $W$ (rather than with center $P$, as we have done earlier in connection with Question B). The pair $\boldsymbol{A}_{n+1} \supset W$ is transformed into a pair $M_{n+1}^{\prime} \supset E_{n}^{\prime}$ of nonsingular varieties of dimension $n+1$ and $n$, where $E_{n}^{\prime}$ is the blow-up of $W$, and $E_{n}^{\prime}$ is fibred by a family of $r$-dimensional projective spaces $F^{\prime}\left(P_{t}\right)$, where $F^{\prime}\left(P_{t}\right)$ is the full inverse image of the variable point $P_{t}$ of $W$. We have the proper transform $V^{\prime}$ of $V$, in $M_{n+1}^{\prime}$, the exceptional variety $\mathcal{E}_{n-1}^{\prime}=E_{n}^{\prime} \cap V^{\prime}$ in $V^{\prime}$ and the fibres $\mathfrak{F}^{\prime}\left(P_{t}\right)=F^{\prime}\left(P_{t}\right) \cap \mathcal{E}_{n-1}^{\prime}$. Since $V^{\prime} \subset M_{n+1}^{\prime}, \mathcal{E}_{n-1}^{\prime} \subset E_{n}^{\prime}$ and $\mathfrak{F}^{\prime}\left(P_{t}\right) \subset F^{\prime}\left(P_{t}\right)$ and since $M_{n+1}^{\prime}, E_{n}^{\prime}$ and $F^{\prime}\left(P_{t}\right)$ are nonsingular varieties, all the three varieties $V^{\prime} \supset \mathcal{E}_{n-1}^{\prime} \supset \mathfrak{F}^{\prime}\left(P_{t}\right)$ all hypersurfaces, locally, at each of their points, and we can therefore consider the equisingularity stratification of each of them. We are interested in particular in the initial fibre $\mathscr{F}^{\prime}\left(P_{0}\right)=\mathscr{F}^{\prime}(P)$. The equisingularity of $V$ at $P$, along $W$, must be reflected somehow in the way the strata of $V^{\prime}$ and $\mathcal{E}_{n-1}^{\prime}$ are re- 
lated to the special fibre $\mathscr{F}^{\prime}(P)$, and also in the way the special reduced fibre $\mathcal{F}^{\prime}(P)$ is related to the general reduced fibre $\mathscr{F}^{\prime}\left(P_{t}\right)$. To illustrate what I have in mind, I shall state a few conjectures which seem reasonable to me, without claiming, however, that these conjectures, even if these were proved to be true, say all that there is to say about the required relationship.

First we introduce the following notation: if $P$ is any point of $V$ then $S(V, P)$ shall denote the (algebro-geometric) stratum of equisingularity of $V$ which contains $P$.

F. If $P^{\prime} \in \mathcal{F}^{\prime}(P)$ and $S^{\prime}=S\left(\varepsilon_{n-1}, P^{\prime}\right)$, then

$$
T\left(S^{\prime}, P^{\prime}\right)+T\left(F^{\prime}(P), P^{\prime}\right)=T\left(E_{n}^{\prime}, P^{\prime}\right) .
$$

G. If $P^{\prime} \in \mathscr{F}^{\prime}(P), S_{1}^{\prime}=S\left(V^{\prime}, P^{\prime}\right)$ and $S_{2}^{\prime}=S\left(\mathcal{E}_{n-1}^{\prime}, P^{\prime}\right)$, then

$$
T\left(S_{1}^{\prime} \cap S_{2}^{\prime}, P^{\prime}\right)+T\left(F^{\prime}(P), P^{\prime}\right)=T\left(E_{n}^{\prime}, P^{\prime}\right) .
$$

H. The (reduced) fibre $\mathcal{F}^{\prime}(P)$ is a specialization of the (reduced) general fibre $\mathscr{F}^{\prime}\left(P_{t}\right)$.

Note that $\mathrm{G}$ implies $\mathrm{F}$.

I shall formulate one final question, whose solution seems to be an essential preliminary for attacking the three questions just formulated above.

If $V$ is equisingular at $P$ along $W$, we call a permissible projection $\pi_{z}$ equisingular if $\Delta_{z}$ is equisingular at $\bar{P}\left(=\pi_{z}(P)\right)$ along $\bar{W}\left(=\pi_{z}(W)\right)$. Let

$$
z_{i}=\sum_{(\nu)} a_{v_{1}, \nu, 2}^{(i)} \cdots, \nu_{n+1} x_{1}^{\nu_{1}} x_{2}^{\nu_{2}} \cdots x_{n+1}^{\nu_{n+1}}, \quad i=1,2, \cdots, n .
$$

I. Prove that there exists a finite set of polynomials $G_{\mu}$ in the indeterminate coefficients $a_{\nu}^{(i)}$ such that $\prod_{\mu} G_{\mu}\left(a_{\nu}^{(i)}\right) \neq 0 \Rightarrow \pi_{z}$ is equisingular.

An affirmative answer to this question would imply, in particular, that if there exist equisingular projections then there also exist transversal equisingular projections, and the existence of these latter projections is essential for an inductive approach to Questions F, G, $\mathrm{H}$.

I will conclude with the following remark. A very special case of equisingularity is analytic triviality of $V$ at $P$, along $W$. That case arises if, for a suitable choice of the local coordinates $x_{1}, x_{2}, \cdots, x_{n}, x_{n+1}$ at $P$, the equation of $W$ is $x_{1}=x_{2}=\cdots=x_{r+1}=0\left(\right.$ where $\left.r=\operatorname{cod}_{V} W\right)$ and the variables $x_{r+2}, \cdots, x_{n+1}$ do not occur in the equation $f=0$ of $V$. I am now making the following assumption: there exists an equisingular projection $\pi_{z}$ of $V$ such that the critical variety $\Delta_{z}$ is analytically trivial at $\bar{P}$, along $\bar{W}$ (we express this assumption by saying that 
$V$ is equisaturated at $P$, along $W$ ). In that special case of equisingularity I was able to prove that $V$ is also topologically equisingular at $P$, along $W$ (see [15]; this answers, in the affirmative, in this special case, the first part of Question E). If, furthermore, the above equisingular projection $\pi_{z}$ is also transversal, then I can also prove that $V$ is differentially equisingular at $P$, along $W$ (unpublished). I may say, quite generally, that the special case of equisingularity, represented by equisaturation, provided me with a great deal of experimental material which will be useful in the study of the general theory of equisingularity.

\section{REFERENCES}

1. S. Abhyankar, Local uniformization on algebraic surfaces over ground fields of characteristic $p \neq 0$, Ann. of Math. (2) 63 (1956), 491-526; Correction, ibid. (2) 78 (1963), 202-203. MR 17, 1134; MR 27 \#145.

2. - Resolution of singularities of arithmetical surfaces, Proc. Conf. Arithmetical Algebraic Geometry (Purdue University, 1963), Harper \& Row, New York, 1965, pp. 111-152. MR $34 \# 171$.

3. - Resolution of singularities of embedded algebraic surfaces, Pure and Appl. Math., vol. 24, Academic Press, New York, 1966. MR 36 \#164.

4. H. Hironaka, Resolution of singularities of an algebraic variety over a field of characteristic zero. I, II, Ann. of Math. (2) 79 (1964), 109-326. MR 33 \#7333.

5. - Normal cones in analytic Whitney stratifications, Inst. Hautes Etudes Sci. Publ. Math. No. 36 (1969), 127-138.

6. J. Milnor, Singular points of complex hypersurfaces, Ann. of Math. Studies, no. 61, Princeton Univ. Press, Princeton, N. J.; Univ. of Tokyo Press, Tokyo, 1968. MR 39 \#969.

7. R. Thom, Ensembles et morphismes stratifies, Bull. Amer. Math. Soc. 75 (1969), 240-284. MR 39 \#970.

8. $\mathrm{H}$. Whitney, Local properties of analytic varieties, Differential and Combinatorial Topology (A Symposium in Honor of Marston Morse), Princeton Univ. Press, Princeton, N. J., 1965, pp. 205-244. MR 32 \#5924.

9. - - Tangents to an analytic variety, Ann. of Math. (2) 81 (1965), 496-549. MR 33 \#745.

10. O. Zariski, The reduction of the singularities of an algebraic surface, Ann. of Math. (2) 40 (1939), 639-689. MR 1, 26.

11. - Local uniformization on algebraic varieties, Ann. of Math. (2) 41 (1940), 852-896. MR 2, 124.

12. - Simplified proof for the resolution of singularities of an algebraic surface, Ann. of Math. (2) 43 (1942), 583-593. MR 4, 52.

13. - Reduction of the singularities of algebraic three dimensional varieties, Ann. of Math. (2) 45 (1944), 472-542. MR 6, 102.

14. - Studies in equisingularity. I. Equivalent singularities of plane algebroid curves, Amer. J. Math. 87 (1965), 507-536. MR 31 \#2243.

15. - - Studies in equisingularity. II. Equisingularity in codimension 1 (and characteristic zero), Amer. J. Math. 87 (1965), 972-1006. MR 33 \#125.

16. - Studies in equisingularity. III. Saturation of local rings and equisingularity, Amer. J. Math. 90 (1968), 961-1023. MR 38 \#5775. 